Instituto Internacional de Investigación y Desarrollo Tecnológico Educativo INDTEC, C.A.

DOI: https://doi.org/10.29394/Scientific.issn.2542-2987.2019.4.E.5.84-94

OAI-PMH: http://www.indteca.com/ojs/index.php/Revista Scientific/oai

Artículo Original / Original Article

\title{
Segregación de las mujeres en rubros tecnológicos
}

Autores: Katihuska Tahiri Mota Suárez Universidad Miguel de Cervantes, UMC motakt@gmail.com

Talca, Chile http://orcid.org/0000-0002-4108-957X

José Andrés Farías Verdugo Universidad Miguel de Cervantes, UMC jose.farias@profe.umcervantes.cl

Talca, Chile https://orcid.org/0000-0003-3644-2698

\section{Resumen}

La desigualdad de género es un gran problema en la dimensión laboral y ha estado presente sin erradicarse de la sociedad. Trabajos tecnológicos, albergan una gran cantidad de machismo entre sus empleados que rechazan en su mayoría que la mujer está siendo afectada por este inconveniente. Estudios sujetan que las empresas a nivel mundial tienen un alto porcentaje de hombres en rubro, mientras que la población femenina no ocupa más que una minoría en este puesto. Además, los sueldos entre hombres y mujeres, históricamente, han tenido una gran diferencia; siendo así la brecha salarial un problema relacionado. Inicialmente surge la pregunta: ¿por qué las mujeres son segregadas de rubros en áreas de la tecnología? Para responder la pregunta, se realizó un estudio descriptivo con metodología cualitativa. Como conclusión se obtuvo que las empresas deben tomar conciencia sobre esta problemática para así impedir la segregación laboral de la mujer y disminuir (con fin de erradicar) la gran brecha salarial que destaca entre hombres y mujeres ya que el trabajo que realizan las mujeres en sus rubros es igual y aún mejor que el trabajo que realizan sus pares masculinos en las mismas empresas.

Palabras clave: tecnología; mujer; empresa.

Cómo citar este artículo:

Mota, K., \& Farías, J. (2019). Segregación de las mujeres en rubros tecnológicos. Revista Scientific 4(Ed. Esp.), 84-94, e-ISSN: 2542-2987. Recuperado de: https://doi.org/10.29394/Scientific.issn.25422987.2019.4.E.5.84-94

Fecha de Recepción:

06-05-2019
Fecha de Aceptación:

12-07-2019
Fecha de Publicación: 05-09-2019 


\title{
Segregation of women in technological areas
}

\begin{abstract}
Gender inequality is a big problem in the labor dimension and has been present without eradicating society. Technological works, house a large number of maleness among its employees who mostly reject that women are being affected by this inconvenience. Studies show that companies worldwide have a high percentage of men in business, while the female population occupies only a minority in this position. In addition, salaries between men and women have historically made a big difference; Thus the wage gap is a related problem. Initially the question arises: why are women segregated from areas in technology areas? To answer the question, a descriptive study with qualitative methodology was carried out. In conclusion, it was obtained that companies must become aware of this problem in order to prevent the segregation of women from work and reduce (in order to eradicate) the large wage gap that stands out between men and women since the work done by women in their items is the same and even better than the work done by their male peers in the same companies.
\end{abstract}

Keywords: technology; women; enterprises.

Date Received:

06-05-2019
Date Acceptance:

12-07-2019
Date Publication: 05-09-2019 


\section{Introducción}

La desigualdad de género ha estado marcando a las sociedades del mundo durante mucho tiempo. En pleno siglo XXI, se sigue presenciando conductas machistas que no permiten que la brecha de género disminuya, por lo que las mujeres se tienen que adaptar a un sistema opresor para poder surgir. Factores como el trabajo, la educación, la inclusión y la salud, son afectados por la brecha de género.

En la dimensión laboral se puede destacar un gran problema, el cual está sellado por la segregación de mujeres en los rubros de las empresas. Está comprobado que la mujer tiene menor acceso salarial a los empleos tecnológicos y a ser promovidas en ellos. Cuando se habla de brecha salarial, en este artículo se hace referencia específicamente a las actividades que cubren la tecnología, ya sean desarrolladores web, analistas de sistemas informáticos, analistas de seguridad de la información, ingeniera o ingeniero de software, científica o científico de datos, administradores de tecnologías de la información, entre otros rubros.

A diferencia del hombre, la mujer tiene ideas y la capacidad de liderazgo para resolver los problemas económicos de la actualidad, pero la segregación de género, que sigue obstaculizando a las mujeres, es también un obstáculo para el mundo. La mujer tiene un alto impacto en el trabajo. Tal aspecto se ha demostrado con estudios respecto a la productividad empresarial, y aunque la brecha de género ha disminuido durante los últimos años, el rendimiento de empresas y organizaciones no ha experimentado un incremento.

Por ello, este trabajo pretende investigar las causas que inducen a que la mujer sea segregada de las empresas relacionadas con la tecnología, e investigar además sobre los factores que disminuyen la participación femenina en ellas. Este estudio fue de tipo descriptivo, con metodología cuantitativa, para lo cual se revisó literatura apropiada al tema y se generan una serie de conclusiones dando respuesta a la problemática planteada basado en un 
análisis de fuentes electrónicas y libros consultados.

\section{Metodología}

Este artículo se basó en fuentes de internet, utilizando un método descriptivo, bajo el paradigma cuantitativo para la comparación de resultados con los autores consultados, la data se analizó para dar respuesta a una de las problemáticas en la sociedad actual, la segregación de las mujeres en rubros tecnológicos, identificando las posibles causas de la misma y las actitudes que la sociedad toma frente a esta.

Una vez obtenidos los resultados y la revisión de la bibliografía se llevó a cabo la discusión de éstos para darle fundamento al artículo mediante el uso de parámetros estadísticos extraídos de fuentes electrónicas, así como también contrastación teórica.

\section{Resultados}

El estudio test de pensamiento computacional diseño y psicometría general realizado por González-Palencia y Jiménez (2016), constatan que: "la educación secundaria obligatoria las alumnas se sienten menos atraídas por la informática que los alumnos y, además, tienen una autopercepción más baja de eficacia en su capacidad computacional que la que manifiestan sus compañeros varones" (pág. 753).

Un estudio de Booking Holdings (2018), habla sobre la desigualdad de género en la industria tecnológica, según el estudio, la desigualdad comienza en el proceso de selección:

El $68 \%$ de las encuestadas piensa que los paquetes de beneficios que ofrecen las empresas no están adaptados a las mujeres y el $75 \%$ piensa que las ofertas de trabajo del sector suelen estar dirigidas a los hombres. El $46 \%$ de las participantes siente que se las trata de forma diferente en el trabajo debido a su género. Este número llega al $59 \%$ en el caso de las mujeres que tienen puestos de alta gerencia, y al 
$58 \%$ en el de las mujeres que forman parte de consejos directivos (pág. 1).

Por lo tanto, la correlación entre los puestos de responsabilidad y la desigualdad de género aumenta si las mujeres quieren avanzar en su carrera. El $57 \%$ de las mujeres con cargos directivos senior indica que no consiguieron un ascenso porque eligieron a un compañero del sexo opuesto. En cambio, entre las mujeres con puestos junior este porcentaje fue del $37 \%$.

Además, Mujica (2018), señaló en su estudio: “...que aunque la brecha salarial de género se redujo del $20 \%$ al $15 \%$ entre 2005 y 2015 , los sueldos de las mujeres continúan siendo más bajos" (pág. 1). Un análisis hecho a 422 hombres mayores de 18 años de todo el territorio de Chile, reveló que el 73\% de los hombres dice que no hay discriminación de género en su trabajo, en su contraparte, el $74 \%$ de los encuestados cree que hay una desigualdad en los salarios entre géneros.

Asimismo, Sepúlveda y Solar (2016), revelaron que: "sólo el $25 \%$ cree que se trata de un tema relevante en la agenda pública. Más importantes 0 urgentes son, dicen, las pensiones (84\%) la educación (83\%), o el mejoramiento de la salud (68\%) y de la seguridad (68\%)" (pág. 1).

Según datos del Instituto Nacional de Estadísticas en Encuesta Suplementaria de Ingresos, a fines del año 2011, citado por Lara (2014):

La brecha salarial entre hombres y mujeres promedio era de $32,8 \%$, pero subía a $35,4 \%$ entre los trabajadores con educaciones universitaria y al $38,2 \%$ en aquellos con educación secundaria. El sueldo promedio de hombres es de 417.900 pesos mensuales y el de las mujeres es de 280.900 pesos mensuales (pág. 1).

Por otra parte, el Informe de Resultados de la Encuesta Laboral (ENCLA, 2014): se encontró un total de 24.870 empresas feminizadas (aquella empresa cuya composición laboral corresponde a mujeres en un $50 \%$ o más) 
las cuales representan al $27,8 \%$ del total (pág. 20-21); tal como se observa en el gráfico 1.

Gráfico 1. Distribución porcentual de empresas según feminización y trabajadores en ellas. Empleadores (cuestionario autoaplicado).

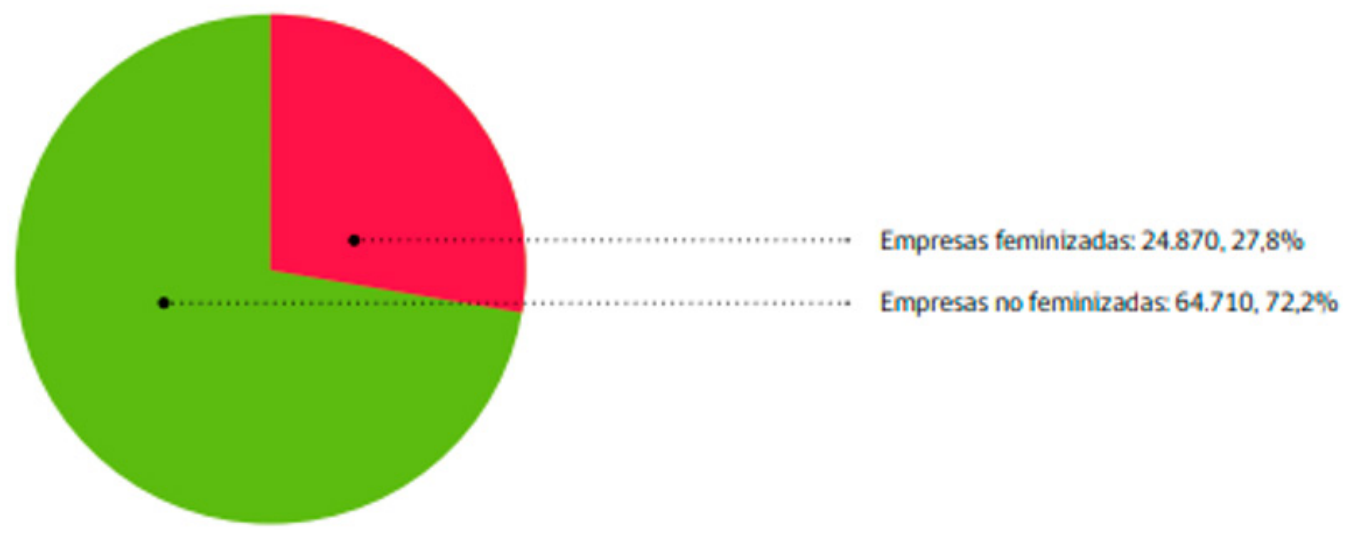

Fuente: ENCLA (2014).

\subsection{Discusión de los resultados}

Los resultados obtenidos en este trabajo mostraron que no solo hay una segregación de género en la vida adulta de las mujeres, sino que desde la infancia y adolescencia ellas son afectadas. Esto se refleja con los resultados del gráfico 1, donde se observa que las jóvenes se sienten menos atraídas por carreras informáticas. Según la Organización para la Cooperación y el Desarrollo Económicos (OCDE), citado por Sanmartín y Matilla (2015):

El problema empieza en edades tempranas, en parte por culpa de los padres y de los profesores. Los progenitores tienen puestas mayores expectativas en los hijos que en las hijas a la hora de apoyarles en sus estudios de Ingeniería o Matemáticas (pág. 1).

De esto se desprende que la educación escolar e intrafamiliar es un determinante importante del sesgo de género en los trabajos relacionados con la tecnología. Por otro lado, se demuestra que uno de los factores de la baja 
participación de las mujeres, se debe a la desigualdad de beneficios y la difícil tarea de poder escalar a un puesto mejor, en especial si se compite con su sexo opuesto, por lo tanto, esta inequidad está tan marcada en la sociedad, que muchas de las mujeres prefieren trabajos que los patrones sociales indiquen que son adecuados para su sexo, como también muchas de ellas migran de estos rubros por las mismas razones.

Es por esto, por lo que la brecha salarial sigue existiendo en el ámbito laboral, no hay una concientización por parte de la sociedad para suprimir estos patrones.

\section{Conclusión}

Si bien se está combatiendo la igualdad de participación entre hombres y mujeres, las empresas tienen un alto grado de responsabilidad en este tema, ya que estas deben aprender a valorar la diversidad y lograr abandonar la antigua sociedad patriarcal en donde todas las acciones ya están escritas para ambos géneros. Si estas tomaran conciencia de esto, muchas de las mujeres capacitadas no abandonarían sus puestos de trabajos por falta de oportunidades o por trato diferente, e incluso por diferencia salarial.

Para concluir, las empresas, en este caso de tecnología, son un reflejo de la sociedad, y es por eso que en la comunidad es muy extraño ver a una ingeniera exitosa, ya que, según la sociedad, ese rubro es de hombres. Muchos de los factores por los cuales las mujeres no son partícipes de rubros en empresas tecnológicas están dados por patrones que la sociedad impone. Estos patrones reprimen las capacidades de mujeres las cuales, pueden ser más eficientes que los hombres en el área ya mencionada.

De acuerdo a la bibliografía consultada, la forma más adecuada para poder erradicar estos tipos de patrones es reflexionando hacia el futuro, ya que es difícil suprimir estos ideales en una persona adulta porque está ya ha vivido toda su vida con ellos. Sin embargo, la esperanza se encuentra en los infantes 
y en las fuentes de información que ellos reciben, como es la educación de los hogares y los colegios, estas son la base para potenciar las habilidades que estos mismos encuentran, sin importar el sexo que ellos tengan, y así poder ir desarrollándose a medida que pasa el tiempo.

No obstante, estas son medidas que tomar hacia futuro, pero es inquietante que esta desigualdad siga existiendo, especialmente en el ámbito laboral, ya que muchas veces esta promueve el abuso e incluso la violencia hacia las mujeres, quienes se encuentran vulnerables en estos rubros. Debido a lo anterior, es fundamental que el gobierno de cada país tome una verdadera responsabilidad sobre esta problemática, implementando políticas que puedan eliminar esta brecha, a modo de regular la cantidad de ingreso de mujeres a empresas tan importantes hoy en día, como son las tecnológicas.

\section{Referencias}

Booking Holdings (2018). Un estudio de Booking.com demuestra que la desigualdad de género en la industria tecnológica va más allá de los campos de la ingeniería y la informática. Madrid, España: Booking.com, B.V. Recuperado de: https://news.booking.com/unestudio-de-bookingcom-demuestra-que-la-desigualdad-de-genero-enla-industria-tecnologica-va-mas-alla-de-los-campos-de-la-ingenieria-yla-informatical

ENCLA (2014). Inequidades y brechas de género en el empleo. Informe de Resultados. Primera Edición, ISBN: 978-956-9661-34-1. Chile: Publicación del Departamento de Estudios de la Dirección del Trabajo. Recuperado de:

http://www.dt.gob.cl/portal/1629/articles-108317 recurso 3.pdf

González-Palencia, R., \& Jiménez, C. (2016). La brecha de género en la educación tecnológica. Ensaio: Avaliação e Políticas Públicas em Educação, 24(92), 743-771, ISSN: 0104-4036; e-ISSN: 1809-4465. 
Recuperado de:

https://dx.doi.org/10.1590/S0104-403620160003000010

Lara, N. (2014). Diferencias de género: ámbito laboral. Chile: elquintopoder; Fundación Democracia y Desarrollo. Recuperado de:

https://www.elquintopoder.cl/genero/diferencias-de-genero-ambitolaboral/

Mujica, F. (2018). Desigualdad de género en el trabajo: Brecha en la participación de hombres y mujeres es de $25 \%$. Santiago, Chile: Empresa El Mercurio, S.A.P. Recuperado de:

https://www.emol.com/noticias/Economia/2018/02/28/896773/Desigual dad-de-genero-en-el-trabajo-Brecha-en-la-participacion-de-hombres-ymujeres-es-de-25.html

Sanmartín, O., \& Matilla, Á. (2015). ¿Por qué las chicas no quieren ser ingenieras? Madrid, España: El Mundo; Unidad Editorial Información General, S.L.U. Recuperado de:

https://www.elmundo.es/espana/2015/07/27/55b025f7268e3e3b6e8b4 $\underline{59 b . h t m l}$

Sepúlveda, P., \& Solar, S. (2016). $\mathbf{7 3 \%}$ de los hombres dice que no hay discriminación de género en su trabajo. Chile: La Tercera, innovación digital. Recuperado de:

http://www2.latercera.com/noticia/73-los-hombres-dice-nodiscriminacion-genero-trabajo/ 


\section{Katihuska Tahiri Mota Suárez \\ e-mail: motakt@gmail.com}

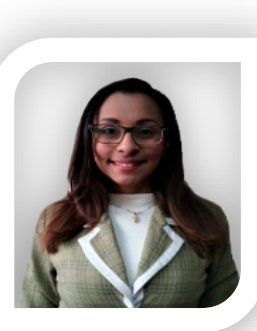

Nacida en Maracaibo, Venezuela, el 11 de abril del año 1982. Docente Académico-Investigador con Doctorado en Ciencias de la Educación titulada en la Universidad Nacional Experimental "Rafael María Baralt", (UNERMB); Magister en Gerencia de Empresas, mención: Operaciones en la Universidad del Zulia (LUZ); Ingeniero de Petróleo titulada en la Universidad del Zulia (LUZ); y Diplomado en Docencia e Investigación en la Universidad del Zulia (LUZ); Con amplia trayectoria como académica a nivel de pregrado y postgrado; asesora de trabajo especial de grado en Cabimas, Estado Zulia, Venezuela; Soy Docente con Promoción al Investigador (PII) categoría A01; Ponente en Conferencias relacionadas con el área de Logística, Matemáticas, Investigación y aspectos relacionados; y Relatora en cursos de liderazgo y motivación. 
José Andrés Farías Verdugo

e-mail: jose.farias@profe.umcervantes.cl

El contenido de este manuscrito se difunde bajo una Licencia de Creative Commons ReconocimientoNoComercial-Compartirlgual 4.0 Internacional 